\title{
An Equilateral Triangle Waveguide Beam Splitter
}

\author{
Zhimin Liu ${ }^{1,2,}$, Fengqi Zhou' ${ }^{1}$, Hongiian $\mathrm{Li}^{2}$, Bin Tang ${ }^{3}$, Zhengfang Liu ${ }^{1}$, \\ Qingping $\mathrm{Wu}^{1}$, Aixi Chen ${ }^{1}$, and Kelin Huang ${ }^{1}$ \\ ${ }^{1}$ School of Basic Sciences, East China Jiaotong University, Nanchang, Jiangxi, 330013, China \\ ${ }^{2}$ College of Physics Science and Technology, Central South University, \\ Changsha, Hunan, 410083, China \\ ${ }^{3}$ Jiangsu Polytechnic University, Changzhuo, Jiangsu, 213164, China \\ liuzhimin2006@163.com
}

\begin{abstract}
In this paper an optical beam splitter based on an equilateral triangle waveguide (ETW) is studied theoretically and numerically. We show that an optical beam splitter bases on ETW formed only when the length of the waveguide and the location of incident light are appropriate. When the length of the ETW is one third of the self-imaging length, the incident light is divided into three identical and symmetrical beams; while the length of the ETW is one nine of the self-imaging length, the incident light is divided into twenty- seven identical and symmetrical beams, if the number is less than twenty-seven, that is because some of images overlap with each other. It is expected that the results obtained here will help to design a new splitter.
\end{abstract}

Keywords: Self-imaging, Equilateral triangle waveguide, beam splitter.

\section{Introduction}

The propagation of light along a waveguide is one of the fundamental and important questions of wave optics. In recent years the splitter and self-imaging of waveguide have been actively discussed. A theoretical and experimental investigation of the selfimaging properties of planar waveguide have been studied [1-3]. Self-images in a rectangular waveguide has been reported [4]. Similar studies [5-6] were performed on square fiber, and it mentioned that round fiber do not have the image transmission characteristics above, that is mainly because of the angular ambiguity and high symmetry. And optical power splitter(OPS) base on multimode interference waveguide has been studied [7-9]. OPS made of photonic crystal waveguide has been studied [10-11]. A few investigators have been discussed the equilateral triangle resonators [12-16] and ray optics model for triangular hollow waveguides [17], but few attentions are paid to investigate the properties of self-images in an ETW. So study on the behavior of light propagation through this waveguide would be of practical interest. In

This work was funded by the National Natural Science Foundation of China (Grant No. 60708014), the Key Natural Science Foundation of Hunan Province (Grant No. 06JJ2034), the Natural Science Foundation of Jiangxi (2008GQW0017) and the Research Foundation of East China Jiaotong University (08JC04,09JC01). 
our previous paper [18], we showed that an image transmission through an ETW, in this paper, we study on the behavior of Gaussian beam propagation(GBP) through this waveguide. An optical beam splitter bases on ETW formed by the new study, the results may have convenience in beam splitter application.

\section{Theory}

As shown in our previous paper [18], consider an ETW with side size $a$ and length $L$ (see Fig.1), and the cladding is a perfect reflector, the field vanish at the boundaries. The field inside the waveguide can be expressed by eigenfunction expansion of Helmholtz equation in the triangular section [19].

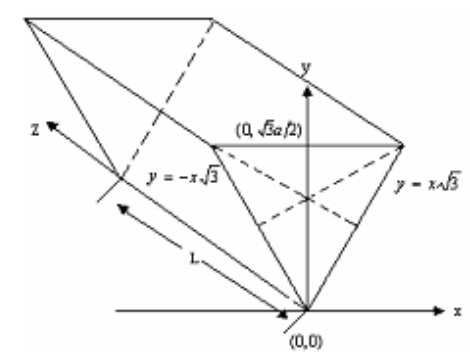

Fig. 1. Schematic diagram of an equilateral triangle waveguide

$$
\begin{aligned}
& E(x, y, z)=\sum_{n=1}^{\infty} a_{(2 n, n)}^{(0)} \psi_{(2 n, n)}^{(0)}(x, y) \exp \left(-i k z \sqrt{1-\frac{4 n^{2}}{3}\left(\frac{\lambda}{a}\right)^{2}}\right) \\
&+\sum_{m 2 n}^{\infty} \sum_{n=1}^{\infty}\left[a_{(m, n)}^{(+)} \psi_{(m, n)}^{(+)}(x, y)+a_{(m, n)}^{(-)} \psi_{(m, n)}^{(-)}(x, y)\right] \exp \left(-i k z \sqrt{1-\frac{4\left(m^{2}+n^{2}-m n\right)}{9}\left(\frac{\lambda}{a}\right)^{2}}\right)
\end{aligned}
$$

For integral values of $m, n$, with the restriction that $m>2 n$. For the case of $m>2 n$, there are two degenerate states with different symmetry properties, which can be written as follows [20] (the correct normalizations are included here):

$$
\begin{aligned}
\psi_{(m, n)}^{(-)}(x, y)=\sqrt{\frac{16}{3 \sqrt{3} a^{2}}\left[\sin \left(\frac{2 \pi(2 m-n) x}{3 a}\right) \sin \left(\frac{2 \pi n y}{\sqrt{3} a}\right)\right.} & -\sin \left(\frac{2 \pi(2 n-m) x}{3 a}\right) \sin \left(\frac{2 \pi m y}{\sqrt{3} a}\right) \\
& \left.-\sin \left(\frac{2 \pi(\mathrm{m}+\mathrm{n}) \mathrm{x}}{3 a}\right) \sin \left(\frac{2 \pi(m-n) y}{\sqrt{3} a}\right)\right]
\end{aligned}
$$

$\psi_{(m, n)}^{(+)}(x, y)=\sqrt{\frac{16}{3 \sqrt{3} a^{2}}}\left[\cos \left(\frac{2 \pi(2 m-n) x}{3 a}\right) \sin \left(\frac{2 \pi n y}{\sqrt{3} a}\right)-\cos \left(\frac{2 \pi(2 n-m) x}{3 a}\right) \sin \left(\frac{2 \pi m y}{\sqrt{3} a}\right)+\cos \left(\frac{2 \pi(\mathrm{m}+\mathrm{n}) \mathrm{x}}{3 a}\right) \sin \left(\frac{2 \pi(m-n) y}{\sqrt{3} a}\right)\right.$ 
In the special case of $m=2 n$ there is a single nondegenerate state for each $n$, and the wavefunction is given by

$$
\psi_{(2 n, n)}^{(0)}(x, y)=\sqrt{\frac{8}{3 \sqrt{3} a^{2}}}\left[2 \cos \left(\frac{2 \pi n x}{a}\right) \sin \left(\frac{2 \pi n y}{\sqrt{3} a}\right)-\sin \left(\frac{4 \pi n y}{\sqrt{3} a}\right)\right]
$$

The coefficients $a_{(2 n, n)}^{(0)}, a_{(m, n)}^{(+)}, a_{(m, n)}^{(-)}$are determined by the projection of the initial distribution onto the waveguide

$$
\begin{aligned}
& a_{(2 n, n)}^{(0)}(x, y)=\iint d x_{1} d y_{1} \psi_{(2 n, n)}^{(0)}\left(x_{1}, y_{1}\right) E_{1}\left(x_{1}, y_{1}\right) \\
& a_{(m, n)}^{(+)}(x, y)=\iint d x_{1} d y_{1} \psi_{(m, n)}^{(+)}\left(x_{1}, y_{1}\right) E_{1}\left(x_{1}, y_{1}\right) \\
& a_{(m, n)}^{(-)}(x, y)=\iint d x_{1} d y_{1} \psi_{(m, n)}^{(-)}\left(x_{1}, y_{1}\right) E_{1}\left(x_{1}, y_{1}\right)
\end{aligned}
$$

In this paper, the initial light we consider a GBP

$$
E_{1}\left(x_{1}, y_{1}\right)=\exp \left[-\frac{x^{2}+(y-\sqrt{3} a / 6)}{\omega_{0}^{2}}\right]
$$

Where $\omega_{0}$ is beam waist size of the GBP, The GBP is launched at $(0, \sqrt{3} a / 6)$, instead of the center $(0, \sqrt{3} a / 3)$, in order to avoid any overlap of the images. The coefficients can't be get analytic solution, which are given by oscillatory numerical integral.

$L_{0}=9 a^{2} / 2 \lambda$ is the self-imaging length of the ETW, the detailed derivation as shown $\mathrm{in}^{[18]}$, that is, at this distance (the self-imaging distance) the initial distribution repeats.

\section{Numerical Results and Analysis}

The output field at the back face of the waveguide was numerically calculated according to Eq.(1) with the waveguide size $a=0.2 \mathrm{~mm}$, the wavelength $\lambda=633 \mathrm{~nm}$ and GBP with $\omega_{0}=10 \mu m$. Modes $m, n$ should be taken on values for all the possible guided-wave modes, in other words, $m^{\max }, n^{\max }$ are determined by

$$
1-\frac{4 n^{2}}{3}\left(\frac{\lambda}{a}\right)^{2} \geq 0 \text { and } 1-\frac{4\left(m^{2}+n^{2}-m n\right)}{9}\left(\frac{\lambda}{a}\right)^{2} \geq 0
$$

But normally, the coefficients decrease rapidly with increasing $m, n$, the actual number of modes which have to taken into account is much less than $m^{\max }, n^{\max }[1]$, in this paper $m, n=100$ are used in calculation.

\subsection{The Field at the Face of $z=0$}

Firstly, at the incident face of the waveguide $z=0$, that is the incident wave. It is clear that the output field consists very well with the input field both in field intensity 
and position from Fig(2). From these we consider that the simulation procedure is correct and feasible.
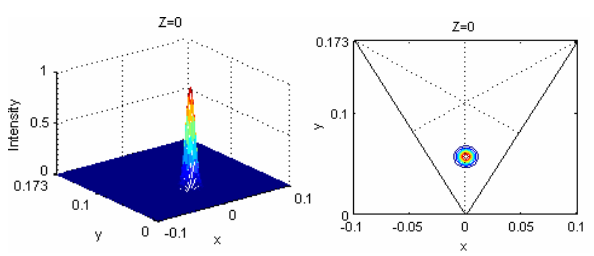

Fig. 2. The field at the distance $z=0$ (right is contour plot)
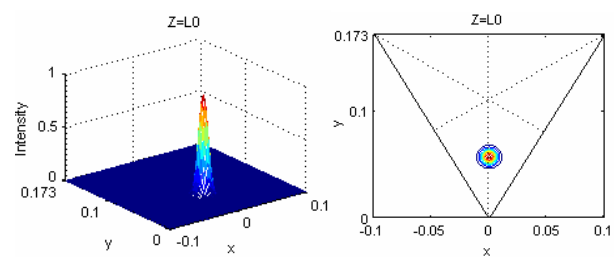

Fig. 3. The field at the distance $z=L_{0}$

\subsection{The Output Field at the Back Face of Self-imaging Length in ETW}

From the theory educing we know $L_{0}=9 a^{2} / 2 \lambda$ is the self-imaging length of the ETW, which means at this distance the initial distribution repeats. Fig(3) demonstrates the result, the field intensity and position of output field consist with the input field. Similar distributions are also observed at the distance $z=n L_{0}$, where $n$ is an integer.

\subsection{The Output Field at the Back Face of Other Length}

As shown in our previous paper [18], similar the results at the distance $z=L_{0} / 3$ are also simulated, obviously the initial distribution is splitted into three identical and symmetrical distributions at the back face of the waveguide, and the output fields have 3 fold rotational symmetry. Moreover, the total intensity of the three beams consists with the intensity of the input field, these are displayed in Fig(4). Fig.5(a) demonstrates the similar results when the input field is launched in the other location, for example the location of $(0, \sqrt{3} a / 4)$.However, if the position of the input field is located in the center of the waveguide $(0, \sqrt{3} a / 3)$, the only one output beam is still located the center, Fig.5(b) shows the result, which is because some of images overlap with each other.
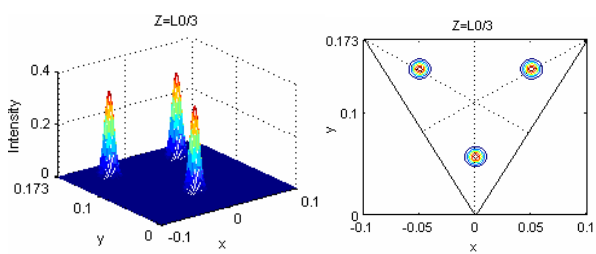

Fig. 4. The field at the distance $z=L_{0} / 3$
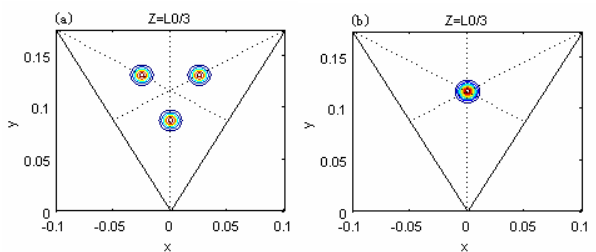

Fig. 5. The incident wave launched at the $(0, \sqrt{3} a / 4)$ and $(0, \sqrt{3} a / 3)$ 
Finally, the field distribution at the distance $z=L_{0} / 9$ are calculated and displayed in Fig(6). The incident light is splitted into nine identical and symmetrical distributions when the incident light is launched at $(0, \sqrt{3} a / 6)$ as shown in Fig.6(c); while the incident light is launched at $(0, \sqrt{3} a / 4)$, the initial wave is splitted into twenty-seven identical and symmetrical distributions, Fig.6(d) shows the result. So the number of the output beams is decided by the location of the incident light. We consider the initial wave can split into twenty- seven at this distance, if the number is less than twenty-seven, that is because some of images overlap with each other. (Note that: Fig.6(d) takes GBP with a waist size of $\omega_{0}=6 \mu m$ to avoid superposition of the field).
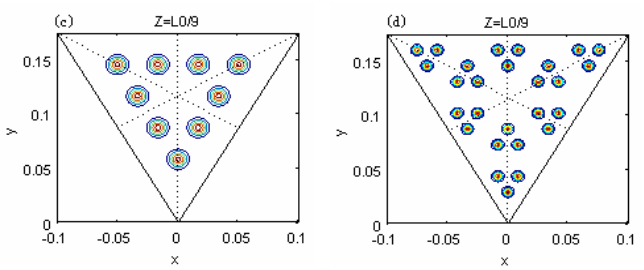

Fig. 6. The field at the distance $z=L_{0} / 9$ : (c) the incident wave launched at the $(0, \sqrt{3} a / 6)$; (d) launched at the $(0, \sqrt{3} a / 4)$

A splitting of the field distributions in waveguide is well known phenomenon, this phenomenon can be used for beam splitters. The main difference between the waveguide beam splitter and other beam splitter is that it can provide many output beams with the same intensity. Another nice property of the ETW beam splitter or the difference between the ETW beam splitter and the other waveguide beam splitter is that the ETW beam splitter can provide $3^{n}(n=0,1,2 \cdots)$ beams with the 3 fold rotational symmetry. So the interesting application of the results as demonstrated in this work is useful in designing a new beam splitters.

\section{Conclusion}

Summarizing, from above discussed, an optical beam splitter bases on ETW formed when the length of the waveguide and the location of identical light are appropriate. When the length of the ETW is one third of the self-imaging length, the incident light is divided into three identical and symmetrical beams; while the length of the ETW is one nine of the self-imaging length, the incident light is divided into twenty- seven identical and symmetrical beams, if the number is less than twenty-seven, that is because some of images overlap with each other. It is expected that the results obtained here will help to design a new splitter. 


\section{References}

[1] Ovchinnikov, Y.B.: Revivals of light in a planar metal waveguide. Optics. Communications $182,35-43(2000)$

[2] Ovchinnikov, Y.B., Pfau, T.: Revivals and oscillations of the momentum of light in a planar multimode waveguide. Physical. Review. Letters 87, 123901 (2001)

[3] Ovchinnikov, Y.B.: A planar waveguide beam splitter. Optics.Communications 220, 229-235 (2003)

[4] He, S.L., Ao, X.Y., Romanov, V.: General properties of NM self-images in a strongly confined rectangular waveguide. Applied. Optics 42, 4855-4857 (2003)

[5] Wu, C.Y., Somervell, A.R.D., Barnes, T.H.: Direct image transmission through a multimode square optical fiber. Optics. Communications 157, 17-22 (1998)

[6] Wu, C.Y., Somervell, A.R.D., Haskell, T.G.: Optical sine transformation and image transmission by using square optical waveguide. Optics. Communications 175, 27-32 (2000)

[7] Sun, Y.L., Jiang, X.Q., Yang, J.Y., Tang, Y., Wang, M.H.: Experimental demonstration of two dimensional multimode-interference optical power splitter. Chinese Physics Letters 20, 2182-2184 (2003)

[8] Han, Z.H., He, S.L.: Multimode interference effect in plasmonic subwavelength waveguides and an ultra-compact power splitter. Optics Communications 278, 199-203 (2007)

[9] Zhang, Y.W., Liu, L.Y., Wu, X., Xu, L.: Splitting-on-demand optical power splitters using multimode interference waveguide with programmed modulations. Optics Communications 281, 426-432 (2008)

[10] Ma, Z.T., Ogusu, K.: Power splitter based on cascaded multimode photonic crystal waveguides with triangular lattice of air holes. Optics Communications 282, 3473-3476 (2009)

[11] Li, W., Xu, X.M.: An ultra-short double-wavelength optical power splitter for two waveguides operation based on photonic crystal multimode interference. Optics Communications 5, 69-73 (2010)

[12] Huang, Y.Z.: Eigenmode confinement in semiconductor microcavity lasers with an equilateral triangle resonator. In: Proceedings-SPIE The international society for optical., vol. 239, p. 3899 (1999)

[13] Guo, W.H., Huang, Y.Z., Wang, Q.M.: Resonant frequencies and quality factors for optical equilateral triangle resonators calculated by FDTD technique and the Padeapproximation. Photonics Technology Letters, IEEE 12, 813 (2000)

[14] Huang, Y.Z., Guo, W.H., Wang, Q.M.: Analysis and numerical simulation of eigenmode characteristics forsemiconductor lasers with an equilateral triangle micro-resonator. Journal of Quantum Electronics, IEEE 37, 100 (2001)

[15] Wysin, G.M.: Resonant mode lifetimes due to boundary wave emission in equilateral triangular dielectric cavities. Journal of optics A: Pure and Applied. Optics 7, 502-509 (2005)

[16] Huang, Y.Z., Guo, W.H., Yu, L.J., Lei, H.B.: Analysis of semiconductor microlasers with an equilateral triangle resonator by rate equations. Journal of Quantum Electronics 37, 1259-1264 (2001)

[17] Isaac, G., Khalil, D.: Ray optics model for triangular hollow silicon waveguides. Applied Optics 45, 7567 (2006) 
[18] Liu, Z.M., Zhu, K.C., Tan, B., Hao, Z.Q., Wen, W.: Image transmission through a mental equilateral triangle waveguide. Chinese Journal of Quantum Electronics 24, 253-256 (2007) (in chinese)

[19] Doncheski, M.A., Robinett, R.W.: Quantum mechanical analysis of the equilateral triangle billiard: periodic orbit theory and wave packet revivals, 8 (2003), arXiv: quant$\mathrm{ph} / 0307063$

[20] Lin, S.L., Gao, F., Hong, Z.P., Du, M.L.: Quantum spectra and classical orbits in twodimensional equilateral triangle billiards. Chinese. Physics. Letter 22, 9-11 (2005) 